\title{
Physicochemical and Biological Characterization of Novel Membrane-Active Cationic Lipopeptides with Antimicrobial Properties
}

\author{
Joanna Juhaniewicz-Dębińska ${ }^{1}$, Robert Lasek², Dagmara Tymecka ${ }^{3}$, Kinga Burdach ${ }^{1}$, Dariusz \\ Bartosik², Stawomir Sęk*1 \\ ${ }^{1}$ Faculty of Chemistry, Biological and Chemical Research Centre, University of Warsaw, Żwirki \\ i Wigury101, 02-089 Warsaw, Poland \\ ${ }^{2}$ Faculty of Biology, Institute of Microbiology, Department of Bacterial Genetics, University of \\ Warsaw, Miecznikowa 1, 02-096 Warsaw, Poland \\ ${ }^{3}$ Faculty of Chemistry, University of Warsaw, Pasteura 1, 02-093 Warsaw, Poland
}

- Number of pages: 9

- Number of tables: 1

- Number of schemes: 1

- Number of figures: 6 


\section{Synthesis of lipopeptides}

Synthesis of Lipopeptides. The preparation of membrane-active lipopeptides utilized solidphase synthesis based on a standard Fmoc/t-Bu methodology. The general route of the synthesis is illustrated in Scheme S1.

Scheme S1. Synthesis of Lipopeptides.

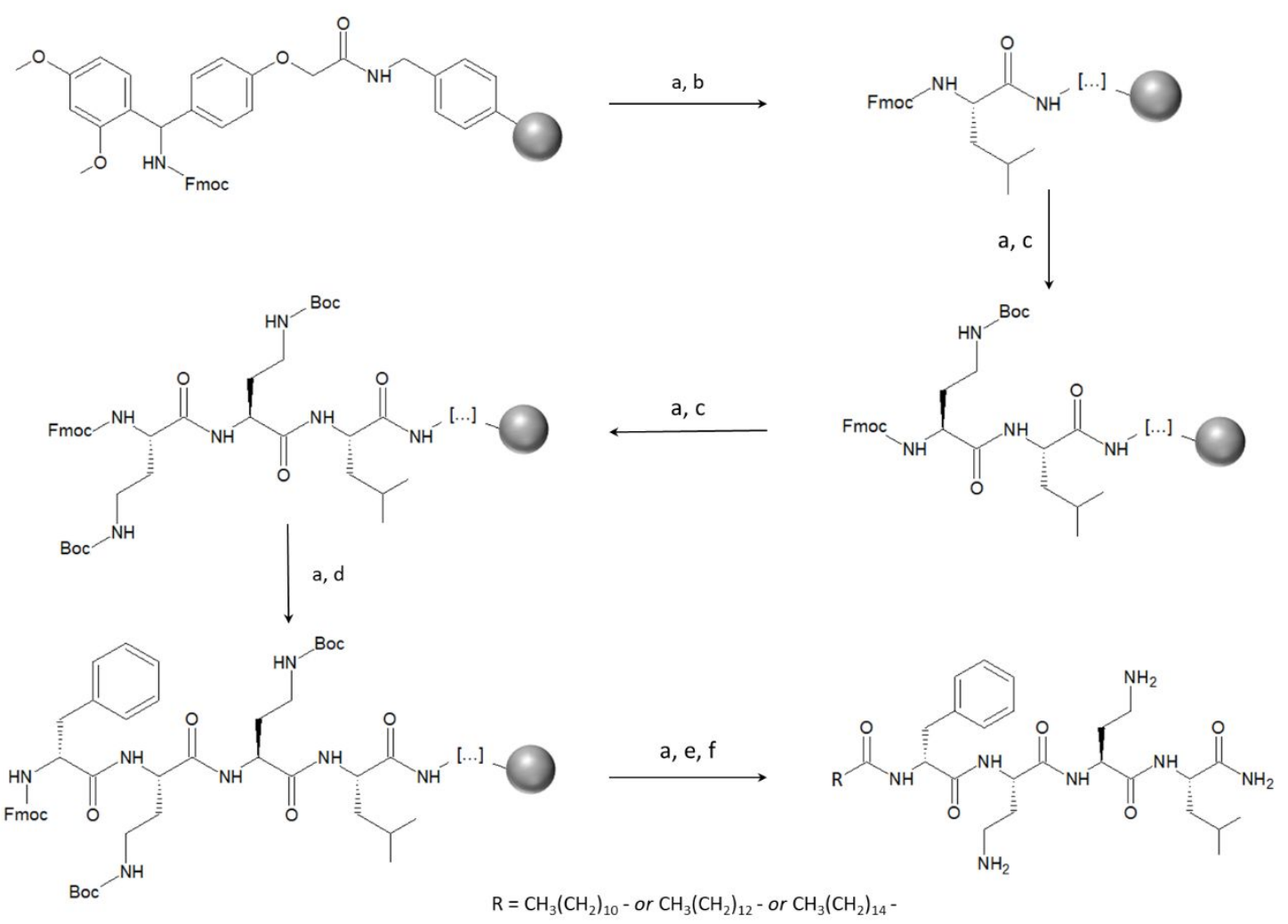

Reagents and conditions: (a) 20\% piperidine in DMF (v/v), rt., 5-10 min; (b) Fmoc-Leu-OH/TBTU/DIPEA, DMF, rt., 4h; (c) Fmoc-Dab(Boc)-OH/ TBTU/DIPEA, DMF, rt., 4h; (d) Fmoc-DPhe-OH/TBTU/DIPEA, DMF, rt. 4h; (e) R-COOH/TBTU/DIPEA, DMF, rt., 24h; (f) TFA/phenol/H $20 / T I P S$ $(88: 5: 5: 2 ; v / v / v / v)$, rt., $4 h$.

We have utilized a standard Fmoc/t-Bu methodology. Rink Amide AM resin with a substitution level of $0.55 \mathrm{mmol} / \mathrm{g}$ and common Fmoc-protected amino acid building blocks in standard Fmoc-Xaa-OH/TBTU/DIPEA protocol (2eq/2eq/4eq) were used. The same procedure was used for coupling the fatty acid to the $\mathrm{N}$-termini of the peptide. The final lipopeptide was cleaved from the resin using Reagent $B$, that is TFA/phenol/ $\mathrm{H}_{2} \mathrm{O} / \mathrm{TIPS}$ (88:5:5:2; v/v/v/v). The product was purified using reversephase HPLC (Shimadzu Prominence system) and C18 Luna column (Phenomenex, 150 $\mathrm{mm} \times 10 \mathrm{~mm}, 5 \mu \mathrm{m}$ ) with a linear gradient of $\mathrm{H}_{2} \mathrm{O}-\mathrm{ACN}-0.1 \%$ TFA. Relevant fractions were lyophilized. The purity of the lipopeptides was estimated from analytical HPLC and in all cases, it was better than $95 \%$. The molecular weight was determined by HRESI-MS. 


\section{Critical micelle concentration (CMC)}
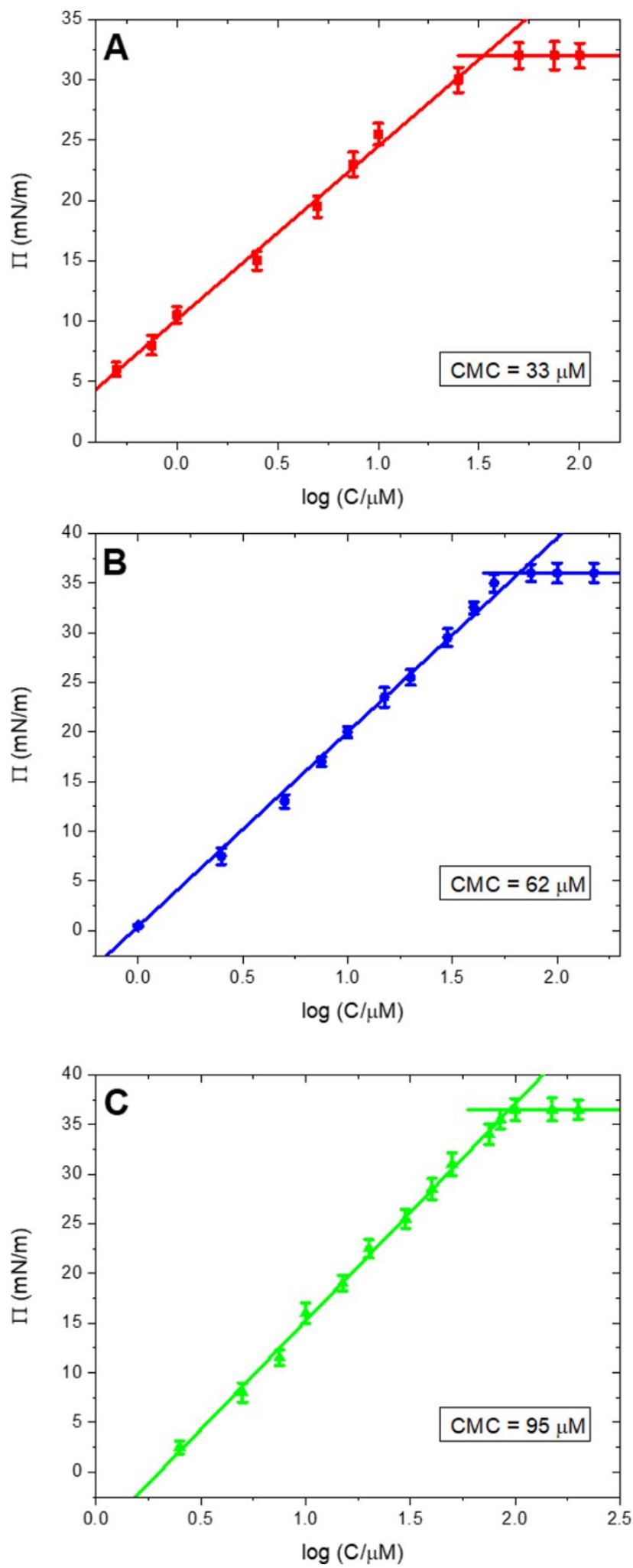

Figure S1. Changes in the surface pressure as a function of the natural logarithm of the bulk concentration of $\mathrm{C}_{16}-\mathrm{fXXL}(\mathrm{A}), \mathrm{C}_{14}-\mathrm{fXXL}(\mathrm{B})$ and $\mathrm{C}_{12}-\mathrm{fXXL}(\mathrm{C})$. 
3. Surface pressure measurements for one-component monolayers of lipopeptides

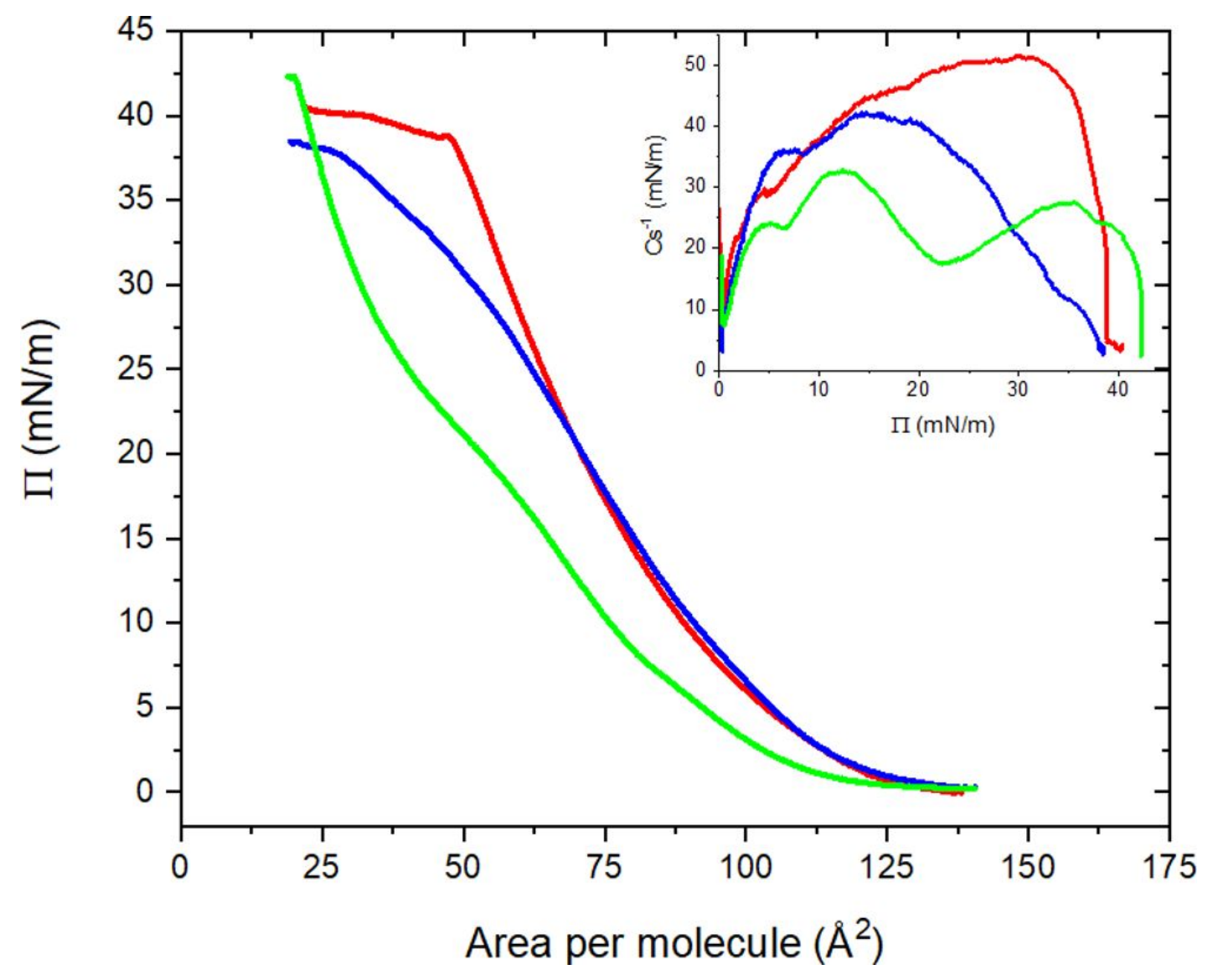

Figure S2. Surface pressure-area per molecule isotherm of $C_{16}-f X X L$ (red), $C_{14}-f X X L$ (blue) and $C_{12}-f X X L$ (green). Inset: compression modulus vs. surface pressure plots. 


\section{Surface pressure measurements for lipid monolayers with lipopeptides}

- reproducibility of the isotherms
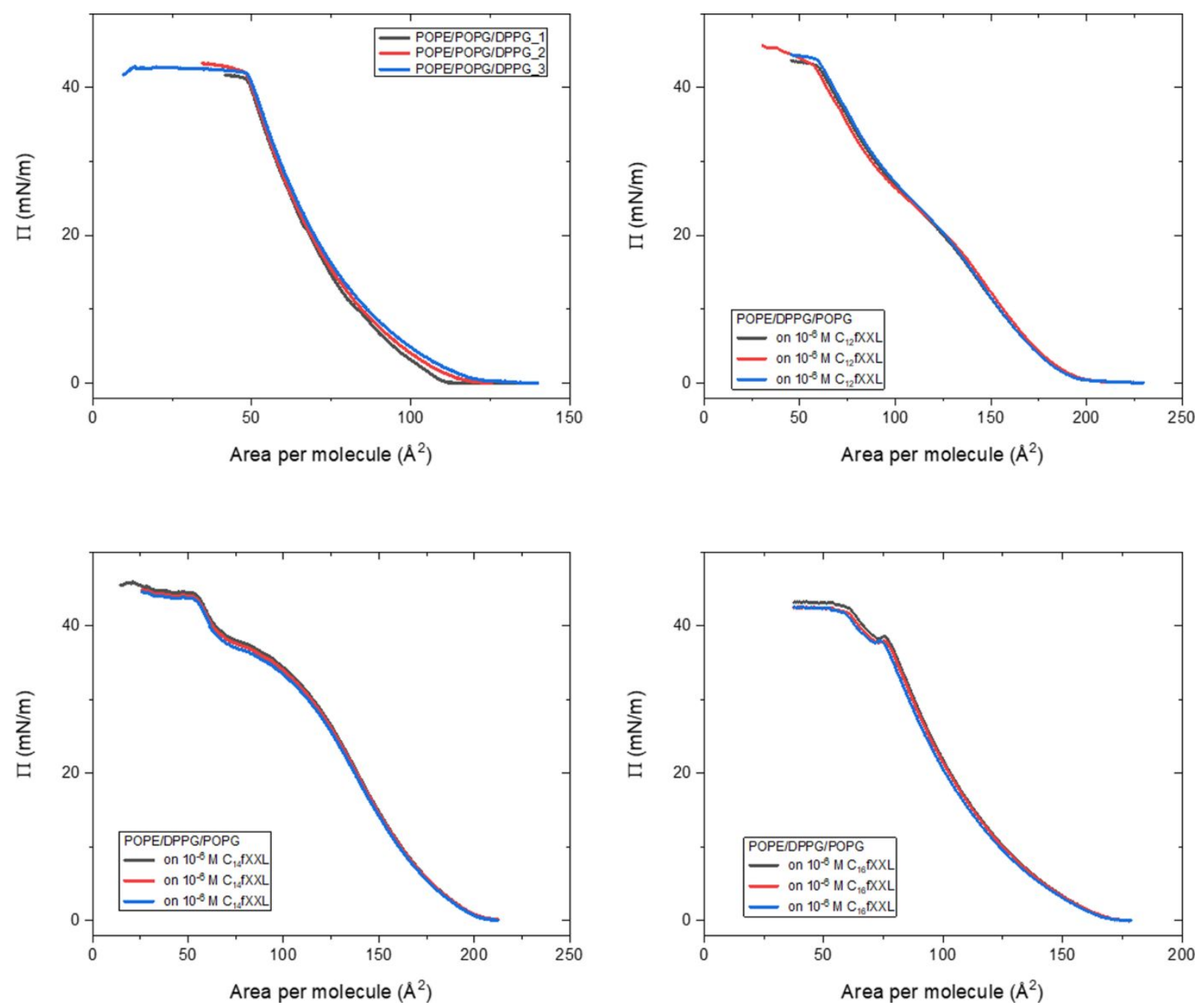

Figure S3. Reproducibility of the surface pressure-area per molecule isotherms recorded for POPE/DPPG/POPG monolayers in the absence and in the presence of lipopeptides. 

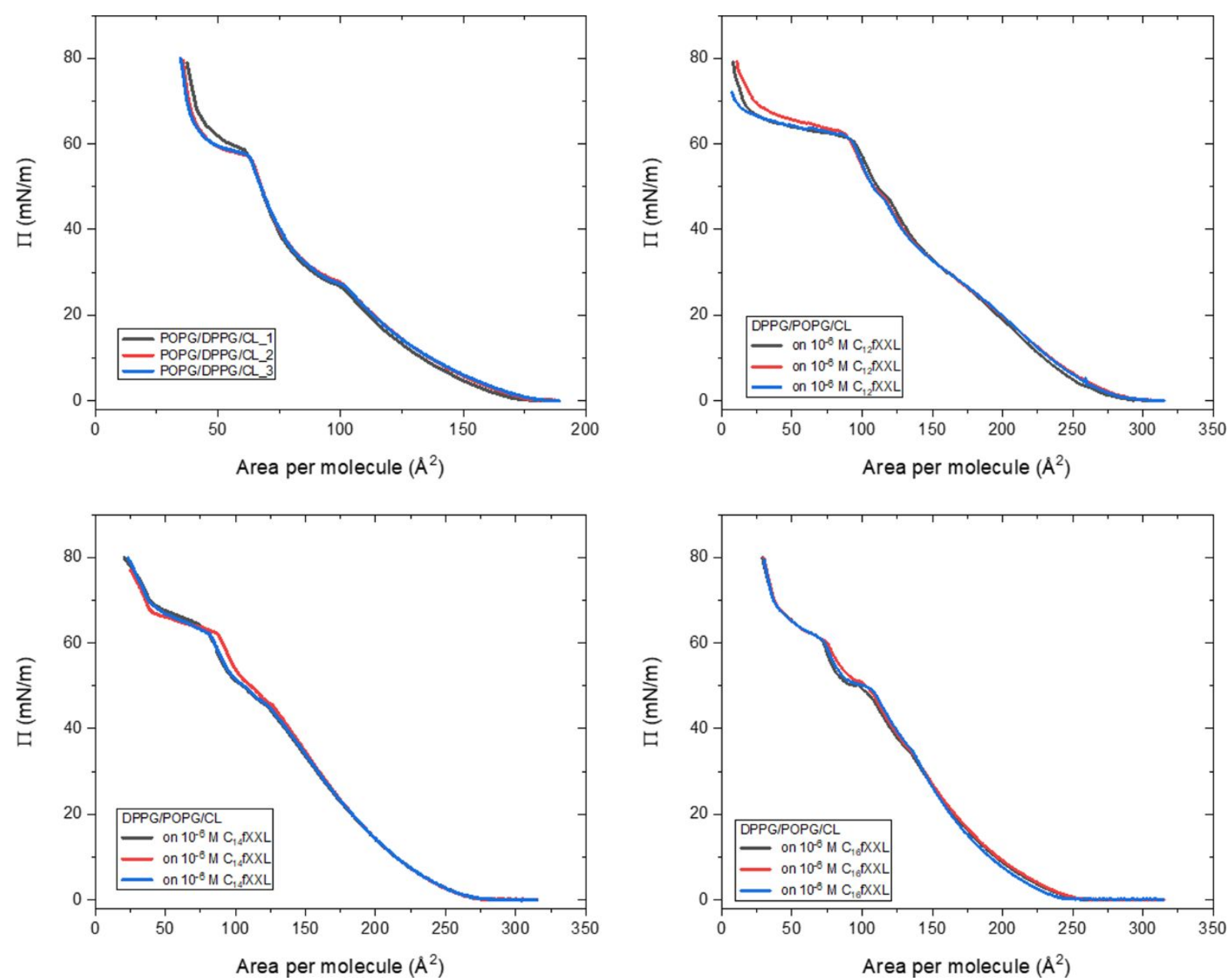

Figure S4. Reproducibility of the surface pressure-area per molecule isotherms recorded for POPG/DPPG/CL monolayers in the absence and in the presence of lipopeptides. 


\section{Surface pressure measurements for lipopolysaccharide monolayers with lipopeptides - insertion of lipopeptides}

To verify whether the lipopeptides can possibly penetrate outer membrane of Gram-negative bacteria, surface pressure measurements were performed using monolayers of lipopolysaccharides (LPS) from Pseudomonas aeruginosa 10, which can be considered as simplified model of the said membrane. The effect of lipopeptides was tested on monolayers pre-compressed at the air-buffer interface. LPS monolayers were firstly compressed to $35 \mathrm{mN} / \mathrm{m}$ and after reaching the target pressure, a stock solution of individual lipopeptide was injected into the subphase under the monolayer to obtain a final concentration of $1 \mu \mathrm{M}$. Then the changes of surface pressure over time were monitored. Exemplary results are shown below:

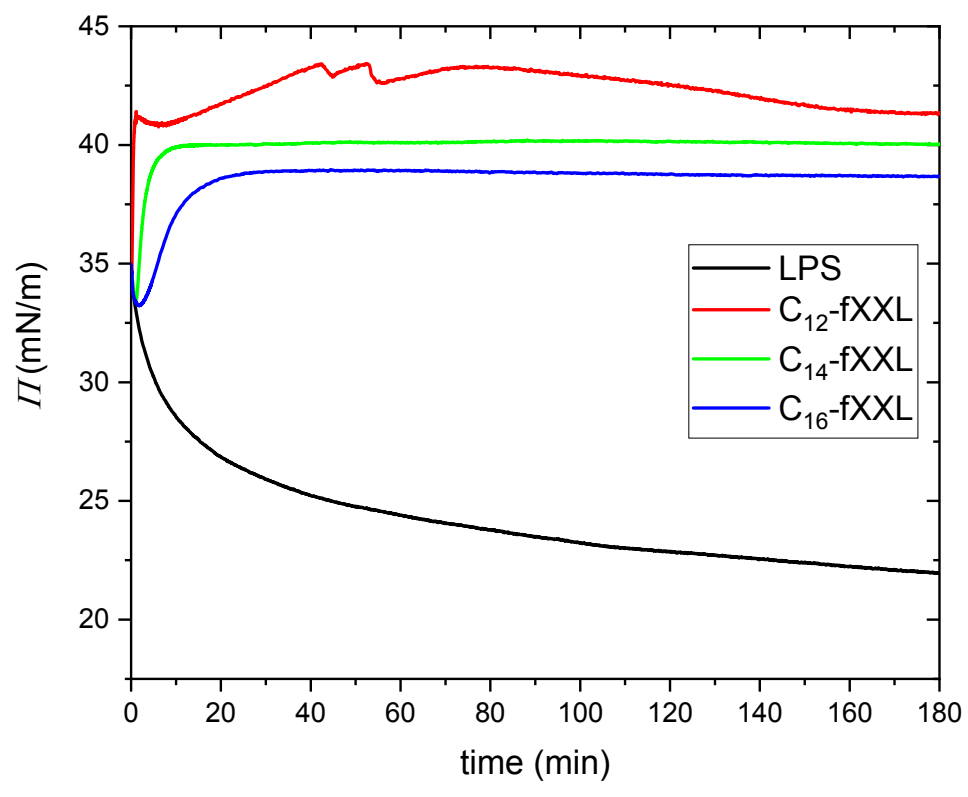

Figure S5. Changes in surface pressure in time recorded for lipopolysaccharide monolayer on pure buffer subphase (black) and after injection of $\mathrm{C}_{12}-\mathrm{fXXL}$ (red), $\mathrm{C}_{14}-\mathrm{fXXL}$ (green) and $\mathrm{C}_{16}-\mathrm{fXXL}$ (blue) into the subphase.

Stability of LPS film is lower compared to lipids. Within the timescale of the experiment, the surface pressure drops from 35 to $\sim 25 \mathrm{mN} / \mathrm{m}$ and then reaches semi-plateau. Nevertheless, the injection of lipopeptides causes noticeable increase of the surface pressure indicative of the insertion of

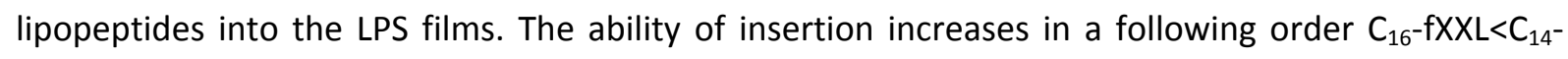
$f X X L<C_{12}-f X X L$. These experimental results imply that lipopeptides can penetrate the outer membrane of Gram-negative bacteria." 


\section{Control experiment with daptomycin}

Bacterial Strains and Growth Media. All tested strains were acquired either from Polish Collection of Microorganisms (PCM) or American Type Culture Collection (ATCC). Grampositive strains tested: Staphylococcus aureus ATCC 29213, Staphylococcus epidermidis ATCC 12228, Gram-negative strain tested (negative control): Escherichia coli PCM 304. All strains were grown in lysogeny broth (LB).

Determination of Minimal Inhibitory Concentration (MIC). A single colony of the tested bacterial strain was transferred to $10 \mathrm{~mL}$ of $\mathrm{LB}$ and grown at $30{ }^{\circ} \mathrm{C}$ overnight. The optical density of the overnight culture was measured and adjusted to be 0.05 (at $600 \mathrm{~nm}$ ) by diluting with LB supplemented with $50 \mathrm{mg} / \mathrm{L} \mathrm{Ca}^{2+}$. Daptomycin was dissolved in DMSO and a dilution series in LB supplemented with $50 \mathrm{mg} / \mathrm{L} \mathrm{Ca}^{2+}$ were made to cover the concentration range from 0.02 to $10 \mu \mathrm{g} / \mathrm{mL}$. The assay was performed by adding $100 \mu \mathrm{L}$ of each of the daptomycin solutions at various concentrations and $100 \mu \mathrm{L}$ of the diluted bacterial culture to the different wells of a 96-well microtiter plate. MIC was defined as the lowest antibiotic concentration required to inhibit the growth of bacteria after $24 \mathrm{~h}$ of incubation at $30{ }^{\circ} \mathrm{C}$ with vigorous shaking (final optical density of $<0.05$ at $600 \mathrm{~nm}$ ). Optical density measurements for the MIC assay were conducted using a TECAN Sunrise plate reader. Data were obtained from five independent experiments.

Determination of the Effect of Daptomycin on Bacterial Growth. The effect of various concentrations of daptomycin on bacterial growth was tested for two Gram-positive strains (S. aureus ATCC 29213, S. epidermidis ATCC 12228) and Gram-negative E. coli PCM 304 as a negative control. It was assessed similarly to MIC, except that the diluted overnight cultures were pre-incubated for 2 hours before the addition of the antibiotic solutions. Subsequently, bacterial growth was monitored by optical density measurements every 90 minutes for $6 \mathrm{~h}$. Data were obtained from four independent experiments.

Table S1. Antimicrobial minimal inhibitory concentrations (MICs) of daptomycin.

\begin{tabular}{ll} 
Bacterial strain & MIC [mg/L] \\
\hline Staphylococcus aureus ATCC 29213 & 4 \\
Staphylococcus epidermidis ATCC 12228 & 4 \\
Escherichia coli PCM 304 & not determined
\end{tabular}



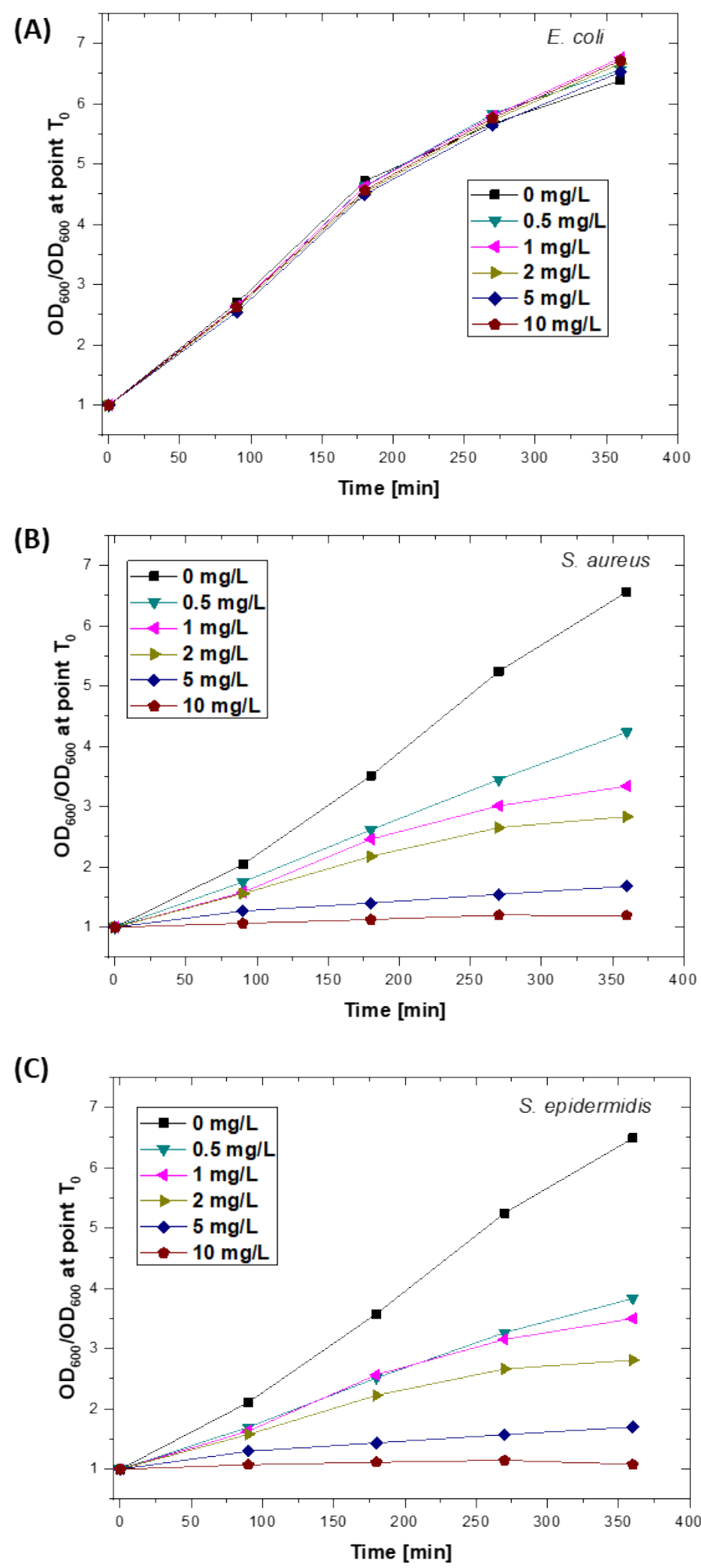

Figure S6. Plots illustrating the changes in optical density at $600 \mathrm{~nm}$ of bacterial culture as a function of time at different concentrations of daptomycin obtained for E. coli PCM 304 (A) S. aureus ATCC 29213 (B); S. epidermidis ATCC 12228 (C) strains. Each data point is the average of 4 measurements. 